\title{
Analyzing Core Migration Protocol Wireless Ad Hoc Networks by Adopting Multiple Nodes Diverse Test-bed
}

\author{
Rahul Malhotra, Reena Aggarwal, Sangeeta Monga \\ Department of Electronics \& Communication Engineering \\ Adesh Institute of Engineering \& Technology, Faridkot (Pb.), India
}

\begin{abstract}
A number of application level multicast protocols have been proposed for core selection and core migration in Mobile Ad Hoc Networks. Core migration is necessary to minimize any disruptions on the transmission of data due to the changes in tree structure and to achieve improvement in the delivery of media streams in multicast group. In this paper, core migration is performed on the varying size network graph model. The nodes within this ad hoc arrangement take on the values of the edge $\left(\mathrm{W}_{\mathrm{e}}\right)$ and node $(\mathrm{W})$ randomly. With the varying numbers of iterations done on the core migration algorithm, every node and every edge picks up different random values. The migration of core node in wireless ad hoc network has been achieved by modeling the network in two different platform independent high-level languages viz. C++ and JAVA. Further, various techniques are proposed for the migration of core if needed.
\end{abstract}

\section{Keywords}

Core-based multicast, mobile ad hoc network, core based tree, core selection, core migration

\section{INTRODUCTION}

In the past few years, a new wireless architecture has been introduced that do not rely on any fixed infrastructure. In this architecture, all nodes are mobile and no node plays any special role. One example of this architecture is the ad hoc mode architecture of 802.11, as shown in Fig.1. In this architecture, 802.11 nodes do not rely on access points to communicate with each other. In fact, nodes reach other nodes they need to communicate with using their neighbors. Nodes that are close to each other discover their neighbors. When a node needs to communicate with another node, it sends the traffic to its neighbors and these neighbors pass it along towards their neighbors and so on. This repeats until the destination of the traffic is reached [6]. Such architecture requires that every node in the network play the role of a router by being able to determine the paths that packets need to take in order to reach their destinations.

Mobile ad hoc network (MANET) is based on rapid deployment of independent mobile users. The routers are free to move randomly and organize themselves arbitrarily thus, the network's wireless topology may change rapidly and unpredictably [1]. Such a network may operate in a standalone fashion, or may be connected to the Internet. Multihop, mobility, large network size combined with device heterogeneity, bandwidth, and battery power constraints [19] make the design of adequate routing protocols a major challenge. Multicast promises efficient use of network bandwidth for multiparty communication by allowing pointto-multipoint communication.

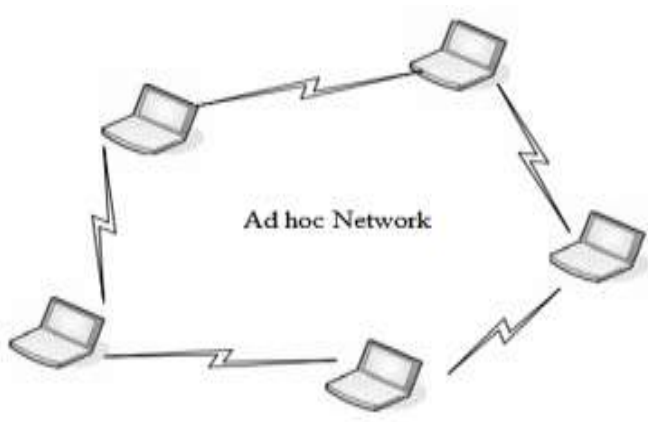

Figure 1. Ad hoc network using IEEE 802.11

Recent [5] multicast protocols such as, Protocol Independent Multicast (PIM) and Core Based Trees (CBT) are based on the notion of group-shared trees. Multicasting routing protocols generally build trees to deliver messages to a multicast group. Delivering the information only through edges belonging to the tree generates an efficient form of multicast communication minimizing the amount of the network resources as required with the unicast routing. In PIM, a group-shared tree is rooted at a rendezvous point (RP) similar to the CBT, which is rooted at the core node. In a multicast tree, the core node maintains the flow of traffic in the network. The [14] selection of this core node influences the shape of the multicast tree influencing the performance of the multicast tree with respect to the amount of the delay experienced by leaves of the tree. Due to the dynamically varying nature of the network topology, nodes need to be migrated to the one which satisfies the specified QoS metrics. Migration of the core node takes place after selecting a core node from the set of candidate core nodes that may be either the neighboring nodes or the nodes adjacent to the neighboring nodes of the previous core node.

In this paper [16] investigate the problem of finding a best node in terms of maximum edge weight (distance between two communicating nodes) and weight of individual node. This implies the movement of the core node on a hop-by-hop basis to reach an optimal location.

The rest of this paper is organized as follows: Section 2 discusses the concept of the core migration in a wireless ad hoc network, the way the nodes join and leave the network. In Section 3, the fundamentals of core migration have been discussed with the different migration techniques. Section 4 shows the results by adopting the multiple nodes.

\section{PROBLEM FORMULATION}

\subsection{Core Based Tree}

The core-based tree protocol is a network layer multicast routing protocol that builds and maintains a shared delivery tree for the multicast routing. Core-based tree is suited to 
inter- and intra-domain multicast routing in the Internet. To establish the path between the sender and the receiver, corebased tree may use a separate multicast routing table, or it may use unicast routing. The core migration protocol followed by the core selection protocol is designed to construct corebased tree. The purpose of the core migration algorithm is to locate that network node or a router whose use as the core of the multicast group results in the best multicast tree with respect to the desired QoS specified by multicast application. The core-based tree is the shortest path tree rooted at some core node. The core node is also referred to as center node or a Rendezvous point [16], [22]. The core-based tree protocol was developed for internet [18]. It uses a shared multicast tree rooted at core node, Rendezvous point or center node. It uses a receiver-based tree construction. A node interested in joining the multicast tree associated with a certain multicast group sends a JOIN-REQUEST towards the core node of that group. The JOIN-REQUEST is routed towards the core node using the underlying unicast routing protocol. An intermediate node receiving a JOIN-REQUEST simply forwards it to the next node in the route towards the core node unless it happens to be an established node in the multicast tree in which case the node responds by sending the JOIN-ACK to the node, which initiated the JOIN-REQUEST. A node traversed by JOIN-ACK record the parent (upstream) node and the child (downstream) node. In effect, the JOIN-ACK grafts a tree branch from the node, which responded with the JOIN-ACK, and the node, which initiated the corresponding JOINREQUEST. A node wishing to leave the multicast group simply sends a QUIT-REQUEST towards the core node along its tree branch. A node receiving a QUIT-REAUEST deletes the node along which it received the QUIT-REQUEST from the children list. Furthermore, it forwards the QUITREQUEST to its parent node if its children list becomes empty and it itself is not a multicast group node.

The CBT protocol provides mechanisms to deal with link/node failures and the core node failure. Link failures are detected by the exchange of periodic keep-alive messages between the neighboring nodes. In case a node does not receive a certain fixed number of consecutive keep-alive messages from its parent node, it assumes that the link to its parent node has failed. In case a non-core node detects that its parent node or link to its parent node has failed, it has two options for failure recovery: it can attempt to rejoin the multicast tree by sending a JOIN-REQUEST towards the core node and it can send a FLUSH-TREE message downstream, thus allowing each node in the sub-tree rooted at it to independently attempt to reattach to the multicast tree [63]. The first option results in the formation of loops in the multicast tree and, hence, the CBT protocol provides a loop detection mechanism.

Core-based multicast routing provides a good mechanism for scalable multicasting. However, migration of a core to an "optimal" location is an essential task. In static networks, core nodes may be pre-assigned and manually configured. However, in mobile ad hoc networks this may not be optimal since the topology often changes. Dynamic selection and hence the migration of center nodes is important for good performance. A good core node may be one, which is at the center of the portion of a network which spans all the multicast of the multicast group, which is the member of the multicast group, and which is fairly stable [18].

This core migration protocol is based on the edge weight of current multicast tree. Core-based multicast tree, as discussed about, have been effectively used in solving the multicast routing problem in mobile ad hoc networks. In this work, this technique has been used to find a core node in which qualitative factor are of major significance with the objective to extract and exploit the goodness of routing and multicast strategies to reach at the best possible route in mobile ad hoc networks. Performance of the core migration method is generally measured using delay as a metric that is the distance of the child (downstream) node from its parent (upstream) node in the entire graph.

\subsection{Network Model}

A mobile ad hoc network can be modeled as a network consisting of $n$ identical mobile hosts (nodes). These mobile hosts employ a packet radio network to communicate with each other. Multiple nodes falling in the radio coverage area of another node are said to be neighbors of this node and they can simultaneously receive a message transmitted from that node. The topology of the mobile ad hoc network is dynamic. Such a network can be represented by an undirected graph $G$ $=\left(\mathrm{V}, \mathrm{E}, \mathrm{W}, \mathrm{W}_{\mathrm{e}}\right)$, where $\mathrm{V}$ is the set of nodes represented $\mathrm{V}=$ $\sum \mathrm{N}_{\mathrm{x}} ; \mathrm{x}=1$ to $\mathrm{n}$, and $\mathrm{E}$ is the set of logical links between neighboring nodes, $\mathrm{W}$ is the weight of each corresponding node in a set of $\mathrm{V}$ nodes, and $\mathrm{W}_{\mathrm{e}}$ represents the weight of the edge connecting the two nodes. It is assumed that nodes leave and join the multicast group arbitrarily.

\subsection{Dynamic Multicast Membership}

Multicast group membership is dynamic, nodes are able to join and leave the group at any time. One of the multicast group members randomly assigns the responsibility of a group leader viz. a core to the first node of a multicast group which then starts broadcasting RREQs. Other group members on receiving RREQs unicasts RREPs along the shortest path to the core. Therefore the nodes are joined to the tree when their corresponding RREPs are accepted either by the existing members or by the core node.

\section{FUNDAMENTALS OF CORE MIGRATION}

\subsection{CORE SELECTION}

Core selection is the problem of selecting the placement of a core or cores in the domain for the purpose of improving the performance of the tree(s) constructed around these core(s). The core selection protocol is designed to construct core based trees using one of the several different QoS metrics. The core selection algorithm tries to find a router whose use as the core of the multicast group results in an optimal multicast tree with respect to a designed performance metric. The selection of the core is based on the explicit as well as implicit core selection algorithms where all network nodes of the multicast group compute their weight functions and exchange weights among themselves so as to select the node with minimum weight as the core for the latter case, and for the former case, the core monitors the delay by calculating the time difference relative to the itself between the transmission of a packet and their corresponding acknowledgements [28]. Core-based multicast routing scheme allows the migration of the core node by selecting the candidate cores (the nodes adjacent to the core node). The selection of the candidate node is based on the heuristic of the comparison between the current core and the set of the adjacent nodes. The node with the significant better performance (in terms of the distance between the adjacent 
nodes ad their individual weight) than the current core is elected as the new core and finally migrated. The migration of the core takes place for the node having largest path delay and minimum node weight, resulting into the optimal core selection and migration. In a typical ad hoc environment, network hosts work in a group to carry out a given task. Multicasting is used for group oriented communications.

Core-based multicast routing scheme allows the migration of the core node by selecting the candidate cores (the nodes adjacent to the core node). The selection of the candidate node is based on the heuristic of the comparison between the current core and the set of the adjacent nodes. The node with the significant better performance (in terms of the distance between the adjacent nodes ad their individual weight) than the current core is elected as the new core and finally migrated. The migration of the core takes place for the node having largest path delay and minimum node weight, resulting into the optimal core selection and migration. In a typical adhoc environment, network hosts work in a group to carry out a given task. Multicasting is used for group oriented communications. [23] discusses the multicast routing based on Centroid Based Core Selection and Random Core Selection techniques.

Centroid Based Core Selection: This core selection method is based on the notion of median node of the current multicast tree. Median of the tree is equivalent to Centroid of tree and proportional to the Bandwidth. The median is calculated as the sum of the weights of all the links in the tree, which signifies the total bandwidth consumed for multicasting a packet.

Random Core Selection: This core selection method selects a core randomly among all the nodes of the graph. In this method, any router in the network, regardless of its own group memberships, can be used as a core node for the group, that is $\mathrm{C}=\mathrm{v}_{\mathrm{i}}$ where $\mathrm{v}_{\mathrm{i}} \in \mathrm{V}$ also $\mathrm{V}$ (number of nodes in a network) is a part of the network model represented as $\mathrm{G}(\mathrm{V}, \mathrm{E}), \mathrm{E}$ as the set of links between the nodes.

Table 1. Bandwidth improvement vs Number of nodes

\begin{tabular}{|l|l|l|l|}
\hline $\begin{array}{l}\text { Number } \\
\text { of nodes }\end{array}$ & $\begin{array}{c}\text { Average BW } \\
\text { with } \\
\text { Random } \\
\text { Core } \\
\text { Selection }\end{array}$ & $\begin{array}{c}\text { Average BW } \\
\text { with } \\
\text { Centroid } \\
\text { based Core } \\
\text { Selection }\end{array}$ & $\begin{array}{c}\text { Improvement } \\
(\%)\end{array}$ \\
\hline 10 & 121.39 & 86.4 & $29 \%$ \\
\hline 20 & 123.37 & 88 & $29 \%$ \\
\hline 30 & 127.32 & 90.4 & $29 \%$ \\
\hline 40 & 134.15 & 94.5 & $30 \%$ \\
\hline 50 & 143.76 & 104.5 & $27 \%$ \\
\hline 60 & 155.785 & 113.5 & $27 \%$ \\
\hline 70 & 169.15 & 121.4 & $28 \%$ \\
\hline 80 & 186.51 & 140.3 & $25 \%$ \\
\hline 90 & 205.345 & 156.2 & $24 \%$ \\
\hline 100 & 223.42 & 168.6 & $25 \%$ \\
\hline
\end{tabular}

It may be seen from these results that centroid based technique is definitely better than the random core selection. For the network sizes varying from 10 to 100 an average improvement over random core selection is $27 \%$, for $110-200$ nodes it is $14.5 \%$, for node sizes $210-300$ it is $12.3 \%, 310-400$ it is $11 \%$, and for $410-500$ nodes average Bandwidth over random core selection technique is $9.8 \%$ respectively.
These core selection techniques used Core-Based tree having a single node, or router, which acts as the core of the tree, which enhances the scalability of the multicast algorithm, also the tree creation was receiver based. The core selection protocol was designed to construct core-based tree so that the core located for the multicast group resulted in the best multicast tree with respect to the desired QoS specified by the multicast application.

Performance of the core selection method was measured using Bandwidth i.e. the sum of all weights from the centroid to the nodes in the entire graph as a metric. Since in this work, the core selection protocol was based on the median of current multicast tree that required minimal information maintenance by each node in the network. In this work two such techniques namely random core selection and centroid based core selection techniques were studied, simulated, and analyzed based on different parameters. These techniques were analyzed by varying node numbers in the network from 10 to 500 in the step of 10 . Further, the link weights between the edges joining these nodes was varied from [0-50] in the step size of [0-5]. It was seen based on the results that centroid based core selection technique proved better than random selection technique and average Bandwidth improvement was above $10 \%$ though it was high as much as $43 \%$ for low number of nodes and as low as $8 \%$ for the tested graph suites. Further it was seen that at small network sizes the improvement is much higher than that at larger network size making this technique more suitable for multicast routing protocols where in normal cases the group size might be limited to nodes less than 100 . The future scope of this work illustrates the migration of the core node under the highly dynamic conditions. To study the core migration, one should be aware of its need.

\subsection{AN APPROACH TO CORE MIGRATION PROTOCOL}

Core migration occurs after selecting a new core for a multicast group. During the lifetime of a multicast application, the members of a group may change, and the resources in the network may fluctuate. The motive of the ore migration is to identify a new core node for the group whose corresponding multicast tree, determined by the current set of group members and present network status, will likely result in significantly better multicast performance than that of the current core [6]. For a fixed network topology, the core migrates to an optimal position on a hop-by-hop basis as opposed to the dynamic network topology where the migration of the core to an optimal position equalizes the edge weight (delay) coming from all the subtree branches [13]. The core migration is based on the core-based multicast routing. This means that, if the performance metrics such as path delay and weight of an adjacent core node is superior to the previously considered node, then that adjacent core will be addressed as the migrated core and the members of the multicast group will then use this migrated core node as their new core node [22]. Typical core-based routing selects one router as a core for a multicast group and builds a single multicast tree rooted at the core to deliver data to the entire multicast group [24].

In this core migration approach, the core determines the edge weight over the several edge weights to find a neighbor experiencing the highest branch weight. The neighbor with the highest branch delay or weight is selected by the core as the 
new core if and only if the final migration to this node will not increase the delay on other branches [14].

TEMP_CORE SELECTION METHOD: Every core maintains an on-route node list (ORNL) provided by the onroute nodes, when the core queries them for their path delays. The on route node list (ORNL) is represented using $\left(\mathrm{N}_{\mathrm{x}}, \mathrm{W}_{\mathrm{e}}\right)$, where $\mathrm{N}_{\mathrm{x}}$ signifies to a particular node among the set of the nodes in a network model and $\mathrm{W}_{\mathrm{e}}$ represents the link weight between the two connecting nodes and serves as the delay in transmission of the data streams. One on route node is chosen from this on-route node list containing the delays received from all these on-route nodes. The selection is primarily based on computing the path delays from the core to all its on-route nodes (all its children nodes). The on-route node with the largest path delay accounts for the largest amount of time required for the transmission of the queries sent and their corresponding replies received. Then it is required to migrate to this on-route node after comparing the weight parameters of the current core with all its children nodes. The migration to the on-route node with largest path delay and minimum weight is desirable.

\subsubsection{Need for the Core Migration in Mobile Ad Hoc Network}

Core Migration can be invoked when the quality of the tree degrades due to membership dynamics or when node/core and/or link failures occur. Due to unconstrained movement of nodes in mobile ad hoc networks, the topology of the network keeps changing. With respect to a multicast tree, a move of a node considers itself as a deletion of a node from the tree followed by addition of the node to the tree. Further, new nodes may join the multicast group and hence the tree [18]. Hence, a node which is a good core node for the multicast tree at a given time may not remain good due to these changes. Hence, a new core node must be periodically chosen. However, changing the core node involves informing all the other nodes of the new core node as well as modifying the multicast tree so that the new core becomes the root of the multicast tree. The core migration in the multicast tree of mobile ad-hoc network is invoked due to [1]:

Recovery from Core Failure: In core-based multicasting, core is a single point of failure. If the core fails, there is a large amount of packet loss as many of the receivers cannot receive the data sent by the senders. Therefore, recovery from both core failure and node/link failure are important issues.

Core Degeneration \& Migration: Due to the dynamic nature of multicast group, core 'degenerates' with time. That is, the quality of the core and hence the multicast tree will deteriorate with time. This means that the core migration has to be invoked when the quality of the core degenerates.

Core migration involves the following: (i) selecting a new core that can offer better performance than the current core, (ii) constructing a multicast tree based on the new core, and (iii)migrating the group members from the current multicast tree to the new multicast tree (tree migration). Thus, core migration plays a vital role in tree maintenance and core failure recovery.

\subsubsection{Core Migration Techniques}

When the membership migrates throughout the network, current routing technique involves selection of a "core" router through which all multicast communication is routed [11]. Then the routing scheme is adapted efficiently to support randomly varying groups by allowing the core node to migrate. In order to evaluate the performance of a core migration method, and therefore that of the resultant multicast trees, the following techniques are generally considered.

\subsubsection{Delay}

It is the distance between the core and the child nodes adjacent to it. The distance of the core from its group members should be minimum for efficient routing. The core monitors the delay of all its adjacent nodes on a hop-by-hop basis. Delay represents the weight of the edge (connecting the two nodes). Therefore, a node with the largest delay is taken for the new core node.

\subsubsection{Weight}

It indicates the unique value assigned to every node in the network. The core node compares its weight with all the neighboring nodes. If the weight of any of the neighboring or the adjacent node is lower than the weight of the core node, then it will stop looking for another node and the node with the lower weight will be considered as the new Core. The core migration is based on the core-based multicast routing. This means that, if the techniques such as path delay and weight of an adjacent core node are superior to the previously considered node, then that adjacent core will be addressed as the migrated core and the members of the multicast group will then use this migrated core node as their new core node Simulations can be done using high level programming using $\mathrm{C}++$ or any another platform.

\subsubsection{Core Migration Algorithm}

1. Core multicast the (Path_Query) message to all its On Route Nodes (viz. to children nodes, here core is the parent node for there ORNs)

2. Then it waits for (Path_Reply) message from all its ORNs.

3. On Route Node List represents the delay between two connecting nodes (here the delays are entered randomly)

$$
O R N L=(\text { received delays from all ORNS })
$$

4. The node with the largest Path Delay is considered as the Temp_Core where it is checked for weight (The weights are entered randomly).

5. Temp_Core then multicast the (Wght_Query) message to all its children.

6. Then it waits for (Wght_Reply) message from all its children.

7. If its own weight is less than lowest adjacent node weight viz. Temp_Core weight $<$ weight of the lowest adjacent node

8. Then migrate the core (viz. now the Temp_Core will be considered as the new core)

9. Else pick the adjacent node with larger delay to be the next Temp_Core, until the node with larger delay and less weight is found. 


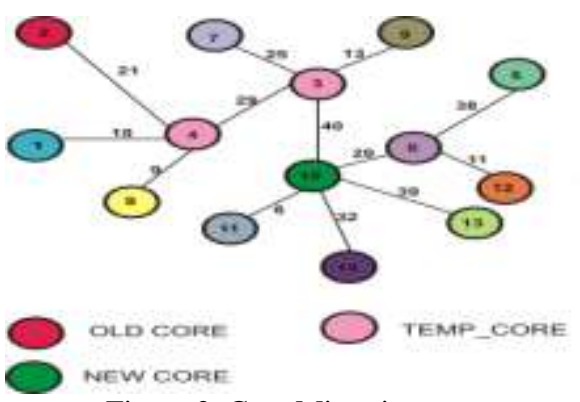

Figure 2. Core Migration

Table 2. Minimum node value for Core Migration

\begin{tabular}{|c|c|c|c|}
\hline $\begin{array}{c}\text { Nodes } \\
\left(\mathbf{N}_{\mathbf{x}}\right)\end{array}$ & $\begin{array}{c}\text { Node Weight } \\
(\mathbf{W})\end{array}$ & Nodes $\left(\mathbf{N}_{\mathbf{x}}\right)$ & $\begin{array}{c}\text { Node Weight } \\
(\mathbf{W})\end{array}$ \\
\hline 1 & 17 & 8 & 16 \\
\hline 2 & 15 & 9 & 16 \\
\hline 3 & 11 & 10 & 5 \\
\hline 4 & 12 & 11 & 7 \\
\hline 5 & 11 & 12 & 11 \\
\hline 6 & 9 & 13 & 9 \\
\hline 7 & 14 & 14 & 7 \\
\hline
\end{tabular}

The algorithm for the migration of the core node in a wireless ad hoc networks given in this section illustrates the node migration based on the two migration techniques discussed above in a 3.2.2. subsection. From Figure 2, it is stated that the core migrates on a hop-by-hop basis by continuously comparing the delay and node values with their child (downstream) nodes, until a node with a minimum node weight and largest delay value appears. Here, the core migration takes place at the $\mathrm{N}_{\mathrm{x}}=10$, as it is having the largest edge value of $\mathrm{W}_{\mathrm{e}}=40$ and the minimum node value of $\mathrm{W}=5$ compared to TEMP_CORE nodes. But if the node value of node 3 is changed to a value of 20 , then the node 4 becomes the finally migrated core.

\section{RESULTS}

The qualitative analysis of the core migration protocol for wireless ad hoc network has been carried out by using a C++ programming model which is further verified by JAVA platform in order to select and migrate the core.

Since the edge weight, the delay between the two adjacent nodes are entered randomly, every edge (connecting link between two nodes) takes on a different values for a number of iterations done. For every network model designed, total of 6 iterations are done to achieve the core migration.

\section{1 Case Study I (using 5 nodes)}

In the first case, the network graph was modeled using $\mathbf{5}$ nodes forming a multicast wireless ad hoc network. All the nodes communicate through mesh structure. When the above mentioned issues related to the wireless ad hoc network appears then core migration algorithm repeats for a number of times, until a best core has been selected and the migrated.

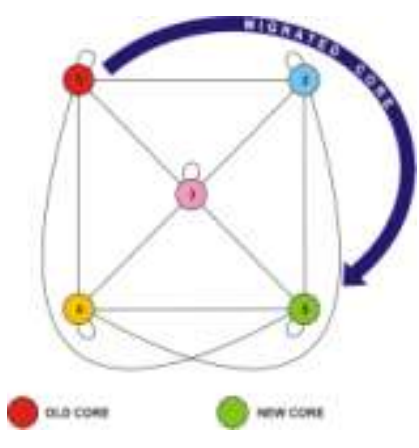

Figure 3. Core Migration using 5 nodes

*TC refers to the TEMP_CORE node of the multicast group.

Table 3. Core Migration using 5 nodes

\begin{tabular}{|c|c|c|c|c|c|c|c|c|}
\hline $\begin{array}{l}\text { No. } \\
\text { of } \\
\text { Itera } \\
\text { tion }\end{array}$ & $\begin{array}{l}\mathrm{T} \\
\mathrm{C} \\
1\end{array}$ & $\begin{array}{l}\text { Del } \\
\text { ay } \\
\text { of } \\
\text { TC } \\
* 1\end{array}$ & $\begin{array}{l}\bar{T} \\
\mathrm{C} \\
2\end{array}$ & $\begin{array}{c}\text { Del } \\
\text { ay } \\
\text { of } \\
\text { TC } \\
2\end{array}$ & $\begin{array}{l}\mathrm{T} \\
\mathrm{C} \\
3\end{array}$ & $\begin{array}{l}\text { Del } \\
\text { ay } \\
\text { of } \\
\text { TC } \\
3\end{array}$ & $\begin{array}{c}\text { Migra } \\
\text { ted } \\
\text { Core }\end{array}$ & $\begin{array}{c}\text { Delay } \\
\text { of } \\
\text { Migra } \\
\text { ted } \\
\text { Core }\end{array}$ \\
\hline 1 & & & & & & & 2 & 13 \\
\hline 2 & 2 & 13 & & & & & 4 & 25 \\
\hline 3 & 2 & 12 & & & & & 4 & 30 \\
\hline 4 & 3 & 10 & 4 & 31 & & & 5 & 40 \\
\hline 5 & 2 & 6 & & & & & 5 & 43 \\
\hline 6 & 2 & 12 & & & & & 5 & 44 \\
\hline
\end{tabular}

In the first iteration scheme, the core gets migrated to the node $2^{\text {nd }}$ being the downstream node with the largest value of delay. The nodes participating in migration process are represented along with their delay values as $\left(\mathrm{N}_{\mathrm{x}}, \mathrm{W}_{\mathrm{e}}\right)$. During the second and the third iteration process, node $4^{\text {th }}$ becomes the new core node with one and only one TEMP_CORE node, which indicates that the participation of the TEMP_CORE decreases with the nodes. During the remaining iteration process, the new core changes to the node $5^{\text {th }}$. For iteration step 4 , the core migration is represented as: $(3,10),(4,31)$, and $(5,40)$.

\subsection{Case Study II (using 7 nodes)}

In the second case, the network graph was modeled using 7 nodes forming a multicast wireless ad hoc network. All the nodes communicate through mesh structure. When the above mentioned issues related to the wireless ad hoc network appears then core migration algorithm repeats for a number of times, until a best core has been selected and then migrated.

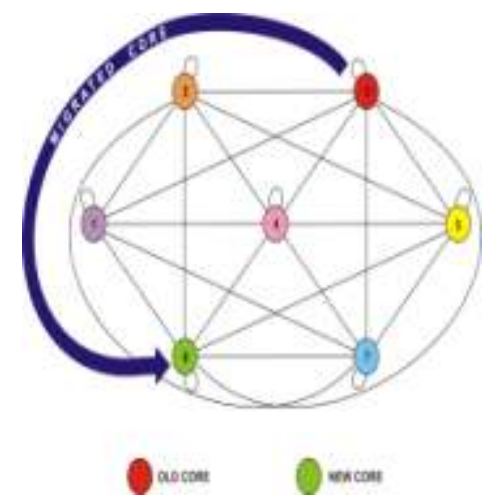

Figure 4. Core Migration using 7 nodes 
During the first iteration scheme, the core gets migrated to the node with the highest value of edge weight viz. the old core is migrated to the node $6^{\text {th }}$ and it becomes the new core node. The new core is selected as the migrated one after passing through the set of TEMP_CORE nodes. During the second iteration process, the migrated core is the same as that of previous iteration scheme but with different number of intermediate nodes participation.

During the fourth, fifth, and sixth iteration process, the migrated core remains stable but with varying values of edge weight. Also the participation by the TEMP_CORE nodes is stable in all these iterations.

Table 4. Core Migration using 7 nodes

\begin{tabular}{|c|c|c|c|c|c|c|c|c|}
\hline $\begin{array}{c}\text { No. } \\
\text { of } \\
\text { Itera } \\
\text { tion }\end{array}$ & $\begin{array}{c}\mathbf{T} \\
\mathbf{C}\end{array}$ & $\begin{array}{c}\text { Del } \\
\mathbf{1} \\
\text { ay } \\
\text { of } \\
\text { TC } \\
\mathbf{1}\end{array}$ & $\begin{array}{c}\mathbf{T} \\
\mathbf{C}\end{array}$ & $\begin{array}{c}\text { Del } \\
\mathbf{2} \\
\text { of } \\
\text { TC } \\
\mathbf{2}\end{array}$ & $\begin{array}{c}\mathbf{T} \\
\mathbf{C}\end{array}$ & $\begin{array}{c}\mathbf{3} \\
\mathbf{D e l} \\
\mathbf{a y} \\
\text { of } \\
\text { TC } \\
\mathbf{3}\end{array}$ & $\begin{array}{c}\text { Migra } \\
\text { ted } \\
\text { Core }\end{array}$ & $\begin{array}{c}\text { Delay } \\
\text { of } \\
\text { Migra } \\
\text { ted } \\
\text { Core }\end{array}$ \\
\hline 1 & 2 & 23 & 4 & 29 & 5 & 31 & $\mathbf{8}$ & $\mathbf{3 2}$ \\
\hline 2 & 2 & 23 & 4 & 31 & & & $\mathbf{5}$ & $\mathbf{3 3}$ \\
\hline 3 & 5 & 12 & 9 & 13 & & & $\mathbf{1 0}$ & $\mathbf{3 5}$ \\
\hline 4 & 5 & 5 & 6 & 18 & 8 & 30 & $\mathbf{1 0}$ & $\mathbf{4 0}$ \\
\hline 5 & 5 & 18 & 8 & 30 & 7 & 33 & $\mathbf{1 0}$ & $\mathbf{4 0}$ \\
\hline 6 & 2 & 19 & 5 & 35 & 3 & 41 & $\mathbf{7}$ & $\mathbf{4 5}$ \\
\hline
\end{tabular}

\subsection{Case Study III (using 10 nodes)}

Finally, the network graph was modeled using 10 nodes forming a multicast wireless ad hoc network.

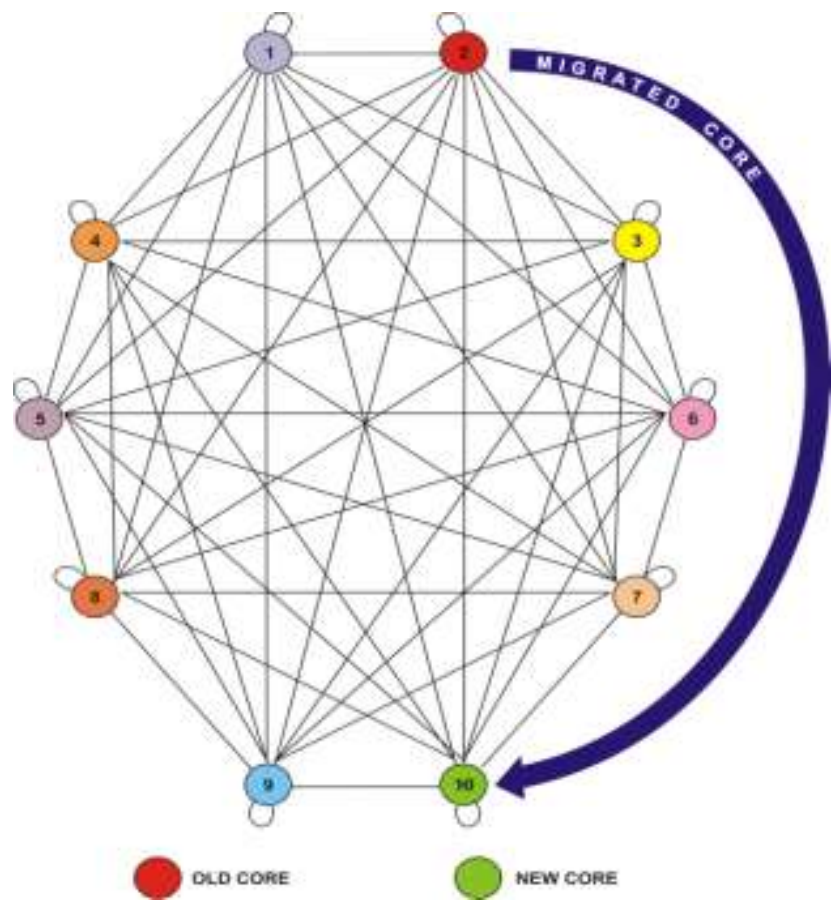

Figure 5. Core Migration using 10 nodes

During the first iteration scheme, the core gets migrated to the node with the highest value of edge weight viz. the old core is migrated to the node $8^{\text {th }}$ and it becomes the new core node. The new core is selected as the migrated one after passing through the set of TEMP_CORE nodes. The TEMP_CORE nodes used in this iteration were $2^{\text {nd }}, 4^{\text {th }}$, and $5^{\text {th }}$ nodes, but

Table 5. Core Migration using 10 nodes

\begin{tabular}{|c|c|c|c|c|c|c|c|c|}
\hline $\begin{array}{l}\text { No. } \\
\text { of } \\
\text { Itera } \\
\text { tion }\end{array}$ & $\begin{array}{l}\mathrm{T} \\
\mathrm{C} \\
\mathbf{1}\end{array}$ & $\begin{array}{c}\text { Del } \\
\text { ay } \\
\text { of } \\
\text { TC } \\
1\end{array}$ & $\begin{array}{l}\mathrm{T} \\
\mathrm{C} \\
2\end{array}$ & $\begin{array}{l}\text { Del } \\
\text { ay } \\
\text { of } \\
\text { TC } \\
2\end{array}$ & $\begin{array}{l}\text { T } \\
\mathbf{C} \\
3\end{array}$ & $\begin{array}{c}\text { Del } \\
\text { ay } \\
\text { of } \\
\text { TC } \\
3\end{array}$ & $\begin{array}{c}\text { Migra } \\
\text { ted } \\
\text { Core }\end{array}$ & $\begin{array}{c}\text { Delay } \\
\text { of } \\
\text { Migra } \\
\text { ted } \\
\text { Core }\end{array}$ \\
\hline 1 & 3 & 2 & 4 & 4 & & & 6 & 31 \\
\hline 2 & 2 & 10 & 4 & 11 & 5 & 20 & 6 & 31 \\
\hline 3 & 3 & 5 & 4 & 11 & & & 5 & 33 \\
\hline 4 & 4 & 11 & 5 & 31 & & & 6 & 34 \\
\hline 5 & 4 & 11 & 5 & 35 & & & 6 & 38 \\
\hline 6 & 4 & 11 & 5 & 35 & & & 6 & 40 \\
\hline
\end{tabular}

were discarded because of the less edge weight value compared to that of new migrated core.

During the second iteration process, the new migrated core is the node $5^{\text {th }}$ with the largest delay value of 33 in comparison to the delay of the TEMP_CORE nodes ( 23 for node $2^{\text {nd }}$ and 31 for node 4 th).

During the third, fourth, and fifth iteration process, the migrated core remains stable but with varying values of edge weight. This time for third iteration, there are two intermediate nodes in comparison to the iteration process of fourth, and fifth. In these three iteration schemes, node $10^{\text {th }}$ is selected as the new migrated core. In the last iteration scheme, the core is migrated to the node $7^{\text {th }}$.

\section{CONCLUSION}

The main objective of this paper was to throw some light on applicational aspect of core selection and core migration in wireless ad-hoc networks. Research in wireless networks is progressing very fast and numerous researchers from various fields focusing to develop some workable scheme to find the best core node or migration of the core if necessary. Core selection in mobile ad-hoc network has studied. Keeping in view, the merits and demerits of centroid-based core selection and random based core selection, this paper suggest the need and possible technique of core migration wherever applicable. The results for the core migration in wireless ad hoc networks obtained with $\mathrm{C}++$ platform are verified with that of the JAVA platform, and it was concluded that the core migration achieved with the JAVA programming results into the optimal location of the core than that obtained from the network graph modeled using $\mathrm{C}++$.

\section{FUTURE WORK}

Further some other parameters except delay and node weight such as hop count, total path cost, throughput, and bandwidth need to be considered in future when migrating the core to a best optimal position.

\section{REFERENCES}

[1] A. Chakrabarti and G. Manimaran, "A case for Scalable Multicast Tree Migration," Global Telecommunications Conference (GLOBECOM 2001), IEEE, Vol.3, pp. 2026 -2030, August 2001. 
[2] A. Chakrabarti and G. Manimaran, "A case for tree migration and integrated tree maintenance in QoS multicasting," Computer Communications, Volume 26, Issue 9, No.2, pp. 1007-1017, June 2003.

[3] A.Dvir and M.Segal, "Placing and Maintaining a Core Node in wireless Ad Hoc Networks," in Wireless Communications and Mobile Computing, Vol.00, pp.114, 2008.

[4] Ayse Karaman and Hossam Hassanein, "Core-Based Approach in Multicast Routing Protocols," 2003.

[5] C.V.Ravishankar et.al, "Distributed Center-Location Algorithms," IEEE Journal on Selected Areas in Communications, Vol.15, No.3, pp.293-303, April 1997.

[6] Eric Fluery, Philip K. McKinley, and Yih Huang, "LCM: A Multicast Core Management Protocol for Link-State Routing Networks," in Proceedings of IEEE International Conference on Communications, Vol.2, pp.1197-1201, June 1998.

[7] Hwa-Chun Lin, Zhe-Hong Lin, "Selection of Candidate Cores for Core-Based Multicast Routing Architectures," in Proceedings of IEEE International Conference on Communications, pp.2662-2666, 2002.

[8] Jean-Francois Macq and Michel X. Goemans, "Tradeoffs on the Location of the Core Node in a Network," Wiley InterScience Vol. 44, Issue: 3 (October 2004) pp. 179 - 186, 2004.

[9] Kenneth L. Calvert, Ellen Witte Zegura, and Michael J. Donahoo, "Core selection methods for multicast routing," in Proceedings of the 4th International Conference on Computer Communications and Networks (ICCCN '95), Las Vegas, Nevada, pp. 638+, September 20-23, 1995

[10] M. Fleury, A. C. Downton, and A. F. Clark, "On the performance and feasibility of multicast core selection heuristics," IEEE Transactions on Parallel and Distributed Systems, vol. 11, no. 11, pp. 164+, 2000.

[11] M. J. Donahoo and Ellen W. Zegure, "Core Migration for Dynamic Multicast Routing," Proceedings of International Conference, Computer Communication and Networks, 1996.

[12] Mario Gerla, Rajive Bagrodia, et.al, "A Performance Comparison Study of Ad Hoc Wireless Multicast Protocols," in Proceedings of 19th Annual Joint Conference of the IEEE Computer and Communications Societies (IEEE INFOCOM 2000).

[13] Manish Kochhal, Sandeep Gupta et.al, "An Efficient Core Migration Protocol for QoS in Mobile Ad Hoc Networks," IEEE International Performance Computing, and Communications Conference, pp.387-391, 2002.
[14] Manish Kochhal, Sandeep Gupta et.al, “QoS-Aware Core Migration for Efficient Multicast in Mobile Ad Hoc Networks," IEEE International Performance Computing, and Communications Conference, October 2002.

[15] Nen-Chung Wang and Si-Ming Wang, "An Efficient Location-Aided Protocol for Mobile Ad Hoc Networks," Proceedings of $11^{\text {th }}$ International Conference on Parallel and Distributed systems, Vol.1, pp. 335-341, July 2005.

[16] Rahul Malhotra and Savina Bansal, "Investigation of Core Selection techniques in multicasting," M.Tech thesis, 2008.

[17] Roger Zimmermann, Rahul Hampole, and Beomjoo Seo, "Distributed Core Migartion in Multicast Peer-to-Peer Streaming," in Proceedings of International Conference on Parallel and Distributed processing techniques and Applications (PDPTA 2004), June 21-24,2004.

[18] S.K.S.Gupta, P.K.Srimani et.al, "Adaptive Core Selection and Migration method for Multicast Routing in Mobile Ad Hoc Networks," IEEE Transactions on parallel and Distributed Systems, Vol.14, No.1, January 2003.

[19] Stephen Mueller, Rose P. Tsang, and Dipak Ghosal, "Multipath Routing in Mobile Ad Hoc Networks: Issues and Challenges," In Performance Tools and Applications to Networked Systems, volume 2965 of LNCS 2004.

[20] Sudarshan Vasudevan, Jim Kurose and Don Towsley, "Design and Analysis of a Leader Election algorithm for Mobile Ad Hoc Networks," Proceedings of $12^{\text {th }}$ IEEE International conference on Network protocols (ICNP), pp. 350-360, October 2004.

[21] Ting-Yuan Wang, Lih-Chyau Wuu, and Shing-Tsaan Huang, "A Scalable Core Migration Protocol for Dynamic Multicast Tree," Journal of Information Science and Engineering, Vol.19, pp.479-501, 2003.

[22] Deering, S., Estrin, D. L., Farinacci, D., Jacobson, V., Liu, C. and Wei, L., (1996), "The PIM Architecture for Wide-Area Multicast Routing," IEEE/ACM Transactions on Networking, 4(2), pp.153-162.

[23] Gerla, M., Chiang, C. C., and Zhang, L., (1999), “Tree Multicast strategies in mobile, multihop wireless networks," In Mobile Networks and Applications, 4 (3) pp. 193-207.

[24] Puthhividhya, W., Tavanapong, W., Tran, M., and Wong, J., (2004), "Distributed Core Selection with QoS Support," In IEEE International Conference on Commumnications, 4, pp. 2132-2137.

\section{Authors}

Rahul Malhotra is currently working as Associate Professor \& Head, Adesh Institute of Engineering \& Technology, Faridkot. His area of interest includes Wireless Communication, Adhoc Networks, and Fuzzy Logic \& Genetic Algorithms. He is a member of Indian Society for Technical Education (ISTE), The Institution of Electronics \& Telecommunication Engineers (India), Institution of Engineers (India).

Reena Aggarwal is pursuing Masters of Technology in Electronics \& Communication Engineering at Adesh Institute of Engineering $\&$ Technology, Faridkot. Her area of interest includes Wireless Communication, Mobile Adhoc Networks.

Sangeeta Monga is currently working as Associate Professor in Electronics \& Communication Engineering at Adesh Institute of Engineering \& Technology, Faridkot. Her area of interest includes Wireless, OFDMA, DSP, and Signal \& System. 